\title{
RESTING HEART RATE VARIABILITY AS A POSSIBLE MARKER OF COGNITIVE DECLINE
}

\author{
Bernhard Grässler', Anita Hökelmann', and Richard Halti Cabral' \\ ${ }^{1}$ Department of Sport Science, Ott-von-Guericke-University Magdeburg, Germany \\ ${ }^{2}$ Tiradentes University, Aracaju, Brazil
}

\begin{abstract}
:
Cognition is a major subject to be addressed nowadays due to the increasing number of cognitively affected people in most societies. Because of a lack of pharmaceutical therapies treating cognitive decline, its indicators should be diagnosed before it becomes prevalent. Scientific evidence indicates a relationship between cognition and the nervous system, especially its autonomic part. Heart rate variability (HRV) as an indicator of the autonomic nervous system functioning has been studied as a biological marker for the evaluation of cognitive performance. Therefore, $\mathrm{HRV}$ is a possible indicator of cognitive impairment. The aim was to provide a systematic literature review about the association between resting HRV and the cognitive performance. Five cognitive functions were analysed separately: executive functions, memory and learning, language abilities, visuospatial functioning, and processing speed. Furthermore, the global cognitive function evaluated with cognitive test batteries was considered as well. An electronic database search was conducted with five databases. Three search fields comprised HRV, cognitive performance, and adult subjects. The final dataset consisted of 27 articles. Significant correlations in each cognitive function were found, except for processing speed, suggesting a positive association between resting HRV and cognitive performance. Mechanisms underlying this association between cardiovascular health and cognition are discussed. For the future, HRV could be used in diagnostics as an indicator of cognitive impairment before symptoms of dementia get apparent. With a timely diagnosis, preventative tools could be initiated at an early stage of dementia.
\end{abstract}

Key words: autonomic nervous system, cognition, dementia diagnosis

\section{Introduction}

Cognition is a prerequisite to participate successfully in social and everyday life. In fact, cognitive functions usually decrease in late life (Bugg, DeLosh, Davalos, \& Davis, 2007; WildWall, Falkenstein, \& Gajewski, 2011) and a very strong decline leads to dementia in older age. One of the biggest health problems in our future is the increasing number of elderly people and concordant increasing number of people with dementia. Medical care of the population is based on early diagnosis, treatment of reversible conditions in an attempt to restore any mental function but, in many cases, it is just a palliative care. So far, neither medications nor drastic interventions can heal or prevent diseases like dementia; except for some evidence pointing out to physical activity (Blondell, HammersleyMather, \& Veerman, 2014). Then, prevention may be a better option.

The heart rate variability (HRV) describes the beat-to-beat variation of RR-intervals. It is a common diagnostic tool to assess the cardiac auto- nomic system and it has been used as a biological indicator for recognizing emotions (Quintana, Guastella, Outhred, Hickie, \& Kemp, 2012). In some other clinical situations, including diabetic neuropathy and congestive heart failure, HRV is used as a non-invasive diagnostic tool as well (Stein \& Kleiger, 1999; Sztajel, 2004). Beside genetic factors (Singh, Larson, O’Donnell, Tsuji, Evans, \& Levy, 2001), the environment (Togo \& Takahashi, 2009), lifestyle (Valentini \& Parati, 2009), neuropsychological factors (Fatisson, Oswald, \& Lalonde, 2016), age also exerts influences on HRV (Almeida-Santos, et al., 2016; Jandackova, Scholes, Britton, \& Steptoe, 2016). Regular physical exercise has positive effects on HRV and can even attenuate the age-induced decline (Hottenrott, Lauenroth, \& Schwesig, 2004; Melo, et al., 2005). In the context of professional sports training, HRV is a popular diagnostic tool to prevent overtraining (Makivić, Nikić, \& Willis, 2013).

In general, a high variation in heart rate reflects a good state of health. McCraty and Shaffer (2015) 
stated that "too little variation indicates age-related system depletion, chronic stress, pathology, or inadequate functioning in various levels of selfregulatory control systems" but too much variation indicates "arrhythmias and nervous system chaos [which] is detrimental to efficient physiological functioning and energy utilization" (Stein, Domitrovich, Hui, Rautaharju, \& Gottdiene, 2005).

The heart-beat variations are the result of a complex interaction between sympathetic and parasympathetic activity (Billman, 2011). Sympathetic activity leads to a reduction of time between heart beats and energy mobilization, whereas parasympathetic activity has the opposite effect and is associated with vegetative and restorative functions. An optimal HRV reflects a balanced state of the autonomic nervous system (Thayer, Yamamoto, \& Brosschot, 2010). The Neurovisceral Integration Model appears to be an important framework for understanding the mechanism underlying the association between HRV and cognition (Kemp, Koenig, \& Thayer, 2017). According to that model, neural structures responsible for affective, cognitive, and physiological regulation are associated with vagally mediated cardiac function, indexed by HRV (Thayer, Hansen, Saus-Rose, \& Johnsen, 2009). Especially the prefrontal cortex is associated, through its connection with the amygdala, with the cardiovascular system (Thayer \& Lane, 2009). Vagally mediated HRV is supposed to be linked with "a set of neural structures that have been implicated in cognitive, especially executive, function" (Thayer, et al., 2009). Therefore, a greater vagally mediated HRV reflects a good prefrontal neural function, leading to better executive functioning.

A lot of different methods to measure the HRV exist (Shaffer, McCraty, \& Zerr, 2014). Due to the supposed relationship between vagally mediated HRV and cognitive performance, the parameters $\mathrm{HF}$ (power in high frequency range, $0.15-0.4 \mathrm{~Hz}$ ), HF nu (HF power in normalized units:

$\mathrm{HF} /[$ Total Power - very low frequency $] * 100)$ and RMSSD (root mean square of successive differences between normal heart beats; in ms) are considered in this review (Shaffer, et al., 2014; Thayer, Åhs, Fredrikson, Sollers III, \& Wager, 2012). HF is also called respiratory band because it corresponds to the respiratory cycle. Changes in heart rate elicited through respiration are known as respiratory sinus arrhythmia (Shaffer, et al., 2014). Parasympathetic influences on the heart rate are prevalent over all possible frequency ranges but sympathetic influences are prevalent only up to $0.15 \mathrm{~Hz}$ (Thayer, et al., 2012). Therefore, the power in low frequency range (LF, $0.04-0.15 \mathrm{~Hz}$ ) cannot clearly be ascribed to either the sympathetic or parasympathetic system and were not considered in this review (Billman, 2013).
Alzheimer's Disease (AD) is an increasing problem for the health care system. Markers that diagnose $\mathrm{AD}$ in preclinical stages are necessary to initiate prevention at an early stage. Beside cognitive performance tests, HRV could be such a diagnostic marker because of the previously mentioned connections between cognition and heart rate regulation. Although the knowledge of this connection has existed for over 150 years (Thayer \& Lane, 2009), research about the association between HRV and cognitive performance only arose about 15 years ago. For using HRV as a marker for mental health, a relationship with the cognitive performance must be confirmed. Thus, the aim of this review is to provide an overview of the existing literature considering the relationship between resting HRV and cognitive performance.

\section{Methods}

\section{Search strategy}

A search request, consisting of three search fields, was implemented to identify all relevant studies. The search fields were connected with AND. The terms within the fields were connected with OR. This procedure guaranteed that at least one term in each field was found by the databases search engines. The first search field comprised terms relating to HRV. The second field comprised terms relating to cognition. The last field specified the subjects' age characteristic to exclude studies with infants or children. This revie wfollowed the guidelines for writing systematic reviews (Moher, Liberati, Tetzlaff, \& Altman, 2009).

\section{Search string}

Search string: "heart rate variability" OR "HRV" OR "heart rate variance" OR "cycle length variability" OR " $R R$ variability" $O R$ "heart period variability" OR "beat-to-beat variation" OR "beatto-beat variability" OR "cardiovascular fluctuation" OR "cardiovascular fluctuations" OR "heart rate oscillation" OR "heart rate oscillations"

\section{AND}

“cognition" OR "cognitive performance" OR "cognitive function" OR "cognitive functions" OR "memory" OR "executive function" OR "executive functioning" OR "executive functions" OR "cognitive state" OR "cognitive impairment" OR "dementia" OR "Alzheimer" OR "cognitive neurodegeneration" OR "cognitive processing" OR "cognitive process" OR "cognitive processes"

AND

"senior" OR "seniors" OR "elderly" OR "adult"

\section{Search process}

The search process was undertaken with electronic databases on August 18, 2017. Five databases 
were searched (Scopus, Pubmed, Web of Science, Cochrane Library, Medline). In Scopus, the search was limited to the categories title, abstract and keywords. Articles were considered as a possible document type and journal as a source type. Title and abstract were used in Pubmed. In the Cochrane Library, the search was undertaken in the categories title, abstract, and keyword, and was limited to trials. No restrictions were possible in Medline and Web of Science. All articles were downloaded in the citation manager Citavi 5 and duplicates were removed.

\section{Inclusion and exclusion criteria}

We considered all age groups except infants and children. Studies with animals were excluded as well. Articles published before 1980 were not considered for this review. Further inclusion criteria for eligible articles were the following:

- Measurement of HRV in resting state (short- or long-term) but not during tasks

- Conduction and presentation of the results of at least one cognitive task or comparison between groups with divergent cognitive states

- Correlation analysis between HRV and cognition or comparison between groups with different HRV levels

- Written in English language

- Full-text available

- Studies that did not include depressive and/or anxiety patients, or patients with psychiatric illnesses

- No drug trials

- Intervention studies if HRV was measured preand post-intervention and compared with cognitive performance

- Duplicates with the same study sample were used only once

- Only published original articles were considered, meaning we did not consider conference papers, reviews, letters, articles in press and notes

- No beta blockers users nor persons with congestive heart failure, myocardial infarction, or cardiac arrhythmias.

All studies, 220 of them, that did not meet these criteria were removed and excluded from further analysis because they did not fulfil the above described criteria for eligibility. The cognitive tasks were classified into the following cognitive functions, based on a report of Levy (1994):

- Executive functions

- Memory and learning

- Language ability

- Visuospatial functioning

- Processing speed

- Global cognitive function.

This classification should ensure a presentation of a broad spectrum of different cognitive abili- ties. The global cognitive function was used as an additional category because some studies presented only the total score of a cognitive test battery (e.g. MMSE) without differentiating between particular cognitive functions. There are only a few cognitive tasks measuring one specific cognitive function. Therefore, we assigned the cognitive tasks to the function that was suggested as the primary cognitive function by the study authors. If no specific function was mentioned, or the task was not described, we looked in the references of the article and assigned it according to these references.

\section{Data extraction}

After reading the abstracts and method sections of the 493 identified articles, 220 articles that did not meet the inclusion criteria were excluded and the full texts of the relevant articles were examined. The cognitive tasks were then classified according to their evaluated cognitive function. Figure 1 describes the search process. The five databases revealed 948 articles. After removing duplicates, 493 remained. Twenty-seven studies met the inclusion criteria and were described in the tables with the following data: first author, year of publication, characteristics of study sample (number of subjects, mean age or age range with standard deviation, if available, and state of health), measured HRV parameters, cognitive tasks, and results.

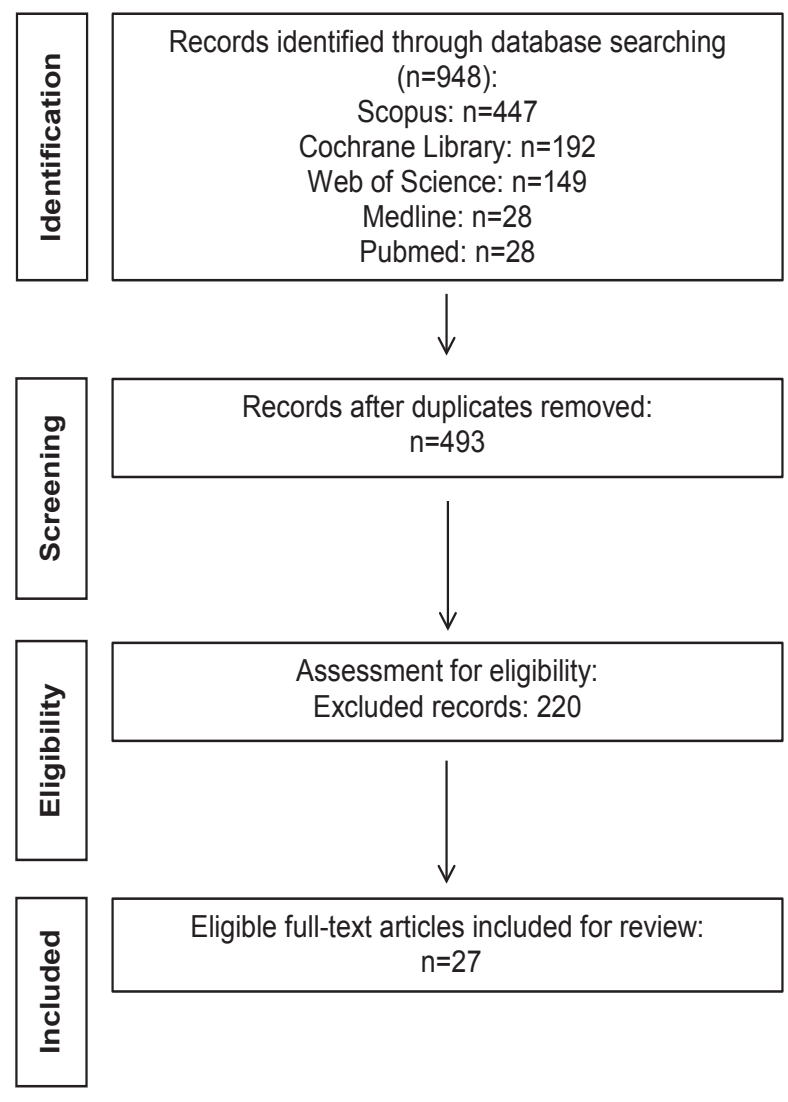

Figure 1. Flow chart of the screening process (n=number of articles) 


\section{Results}

\section{Executive functions}

Table 1 summarizes all studies using executive function tasks. A typical executive task is the task Stroop. Positive correlations were found with HF (Hovland, et al., 2012; Jennings, Allen, Gianaros, Thayer, \& Manuck, 2015), but two other studies found no association with HF (Mathewson, et al., 2010; Nonogaki, Umegaki, Makino, Suzuki, \& Kuzuya, 2017). Stenfors, Hanson, Theorell, and Osika (2016) showed that the association with RMSSD and HF diminished after considering age and sex. Albinet, Abou-Dest, André, and Audiffren (2016) found no association with RMSSD and HF. The study samples comprised young and healthy but also elderly subjects and patients with AD. Therefore, positive relations cannot be limited to specific groups of people.

Two-back is another important executive task. Three studies showed significant associations with HF and RMSSD (Hansen, Johnsen, \& Thayer, 2003; Hansen, Johnsen, Sollers III, Stenvik, \& Thayer, 2004; Hansen, Johnsen, \& Thayer, 2009), but Albinet et al. (2016) and Stenfors et al. (2016) did not find any correlations for the same parameters. WCST (Wisconsin Card Sorting Test) is a further popular executive task. Two studies found positive correlations: Albinet, Boucard, Bouquet, and Audiffren (2010) for RMSSD and
HF, and Hovland et al. (2012) for HF. In the TrailMaking-Test B, positive associations (Kemp, et al., 2016) and no associations (Jennings, et al., 2015; Nicolini, et al., 2014; Stenfors, et al., 2016) with HF were found. Positive correlations were found for the CPT (Continuous Performance Test) with HF and RMSSD (Hansen, et al., 2003, 2004, 2009). A Psychomotor Vigilance Task showed better results in a group with higher RMSSD (Luque-Casado, Zabala, Morales, Mateo-March, \& Sanabria, 2013). HF and RMSSD were positively associated with better results in a Flanker Test (Williams, Thayer, $\&$ Koenig, 2016). However, another version of the Flanker Test was not associated with HF (Alderman $\&$ Olson, 2014). No relationships were detected in some other familiar tasks testing executive functions, e.g. Raven Colored Progressive Matrices (Incalzi, et al., 2009; Nicolini, et al., 2014), d2 (Duschek, Muckenthaler, Werner, \& Reyes del Paso, 2009), Hayling-Test (Albinet, et al., 2016), and Alice-Heim 4-I (Britton, Singh-Manoux, Hnatkova, Malik, Marmot, \& Shipley, 2008). Finally, Yang, Tsai, Hong, Yang, Hsieh, and Liu (2008) detected positive relationships between RMSSD and results of the CASI (Cognitive Abilities Screening Instrument). Some other studies showed no correlations between HRV parameters and executive functions (Frewen, Finucane, Savva, Boyle, Coen, \& Kenny, 2013; Giuliano, Gatzke-Kopp, Roos, \& Skowron, 2017; Kimhy, et al., 2013).

Table 1. HRV parameters and executive functions

\begin{tabular}{|c|c|c|c|c|c|}
\hline Author & Year & $\begin{array}{l}\text { Participants (number/ } \\
\text { age in } M \pm S D \text { or/and } \\
\text { range in years) }\end{array}$ & HRV parameters & Cognitive task & Result \\
\hline Albinet et al. & 2010 & $24 / 70.7 \pm 4.2 ;$ sedentary & RMSSD, HF & WCST & $\uparrow$ \\
\hline \multirow[t]{9}{*}{ Albinet et al. } & 2016 & 36/60-75; sedentary & RMSSD, HF & Stroop & $\leftrightarrow$ \\
\hline & & & RMSSD, HF & Random Number Generation-Test & $\leftrightarrow$ \\
\hline & & & & Hayling-Test & $\leftrightarrow$ \\
\hline & & & & Spatial Running Span-Test & $\leftrightarrow$ \\
\hline & & & & 2-back-Test & $\leftrightarrow$ \\
\hline & & & & Verbal Running Span-Test & $\leftrightarrow$ \\
\hline & & & & Dimension-switching-Test & $\leftrightarrow$ \\
\hline & & & & Plus-Minus-Test & $\leftrightarrow$ \\
\hline & & & & Digit-Letter-Test & $\leftrightarrow$ \\
\hline $\begin{array}{l}\text { Alderman \& } \\
\text { Olson }\end{array}$ & 2014 & $56 / 18-25$; healthy & HF & Eriksen Flanker-Test & $\leftrightarrow$ \\
\hline Britton et al. & 2008 & $\begin{array}{l}\text { 5375/55.5 (men), } 55.8 \\
\text { (women) }\end{array}$ & HF & Alice-Heim 4-I & $\leftrightarrow$ \\
\hline Duschek et al. & 2009 & $60 / 24.5 \pm 3.7$; healthy & HF & d2 & $\leftrightarrow$ \\
\hline Frewen et al. & 2013 & $4763 / 61.7 \pm 8.3$; healthy & HF & MOCA & $\leftrightarrow$ \\
\hline Giuliano et al. & 2017 & $\begin{array}{l}42 \text { women/30.42 } \pm 6.54 \\
\text { stressed }\end{array}$ & HF & visual change detect test & $\leftrightarrow$ \\
\hline \multirow[t]{2}{*}{ Hansen et al. } & 2004 & 37 men/19.1; healthy & HF & CPT: executive functions & $\uparrow$ \\
\hline & & & & 2-back & $\uparrow$ \\
\hline
\end{tabular}




\begin{tabular}{|c|c|c|c|c|c|}
\hline Author & Year & $\begin{array}{l}\text { Participants (numberl } \\
\text { age in } M \pm S D \text { orland } \\
\text { range in years) }\end{array}$ & HRV parameters & Cognitive task & Result \\
\hline \multirow[t]{2}{*}{ Hansen et al. } & 2003 & 53 men/23; healthy & RMSSD & CPT: executive functions & $\uparrow$ \\
\hline & & & & 2-back & $\uparrow$ \\
\hline \multirow[t]{2}{*}{ Hansen et al. } & 2009 & $65 / 23.1$; healthy & $\mathrm{HF}$ & CPT: executive functions & $\uparrow$ \\
\hline & & & $\mathrm{HF}$ & 2-back & $\uparrow$ \\
\hline \multirow[t]{2}{*}{ Hovland et al. } & 2012 & $\begin{array}{l}\text { 36/18-50; panic } \\
\text { disorders }\end{array}$ & $\mathrm{HF}$ & WCST & $\uparrow$ \\
\hline & & & & Stroop & $\uparrow$ \\
\hline Incalzi et al. & 2009 & 54/69.1 $\pm 7.7 ;$ COPD & HF nu & $\begin{array}{l}\text { Raven Coloured Progressive } \\
\text { Matrices }\end{array}$ & $\leftrightarrow$ \\
\hline \multirow[t]{4}{*}{ Jennings et al. } & 2015 & $440 / 43 \pm 7.3$; healthy & $\mathrm{HF}$ & Stroop & $\uparrow$ \\
\hline & & & & WMS II + III: SP-BACK, DGT-BACK & $\leftrightarrow$ \\
\hline & & & & DIGVIG1+2 & $\leftrightarrow$ \\
\hline & & & & Trail-Making Test B & $\leftrightarrow$ \\
\hline Kemp et al. & 2016 & $8114 / 51.17 \pm 8.81$; healthy & RMSSD, HF & Trail-Making Test B & $\uparrow$ \\
\hline \multirow[t]{4}{*}{ Kimhy et al. } & 2013 & $817 / 57.11 \pm 11.15 ;$ healthy & $\mathrm{HF}$ & Digits backward span & $\leftrightarrow$ \\
\hline & & & & Category fluency & $\leftrightarrow$ \\
\hline & & & & Number series & $\leftrightarrow$ \\
\hline & & & & SGST & $\leftrightarrow$ \\
\hline \multirow[t]{3}{*}{$\begin{array}{l}\text { Luque-Casado } \\
\text { et al. }\end{array}$} & 2013 & $\begin{array}{l}26 \text { men/19.5; fit \& unfit } \\
\text { group }\end{array}$ & RMSSD & Psychomotor vigilance task & $\uparrow$ \\
\hline & & & RMSSD & Temporal orienting task & $\leftrightarrow$ \\
\hline & & & RMSSD & Duration discrimination task & $\leftrightarrow$ \\
\hline $\begin{array}{l}\text { Mathewson et } \\
\text { al. }\end{array}$ & 2010 & 76/30.6; healthy & $\mathrm{HF}$ & Pictorial Stroop & $\leftrightarrow$ \\
\hline \multirow[t]{6}{*}{ Nicolini et al. } & 2014 & $80 / 78.6 ; 40 \mathrm{MCl}, 40 \mathrm{CG}$ & HF nu & digit cancellation and bell test & $\leftrightarrow$ \\
\hline & & & & digit span backward & $\leftrightarrow$ \\
\hline & & & & Trail-Making Test B & $\leftrightarrow$ \\
\hline & & & & Weigl's colour-form sorting test & $\leftrightarrow$ \\
\hline & & & & $\begin{array}{l}\text { Raven's coloured progressive } \\
\text { matrices }\end{array}$ & $\leftrightarrow$ \\
\hline & & & & Letter fluency & $\leftrightarrow$ \\
\hline \multirow[t]{5}{*}{ Nonogaki et al. } & 2017 & 78/77.1 $\pm 6.2 ; A D$ & $\mathrm{HF}$ & Category fluency test & $\leftrightarrow$ \\
\hline & & & & Letter fluency test & $\leftrightarrow$ \\
\hline & & & & Wechsler: digit symbol subtest & $\leftrightarrow$ \\
\hline & & & & Clock drawing test & $\leftrightarrow$ \\
\hline & & & & Stroop & $\leftrightarrow$ \\
\hline \multirow[t]{4}{*}{ Stenfors et al. } & 2016 & $\begin{array}{l}119 / 47.98 \pm 10.49 ; 25-66 \text {; } \\
\text { healthy }\end{array}$ & RMSSD, HF & 2-back & $\leftrightarrow$ \\
\hline & & & & Trail-Making Test B & $\leftrightarrow$ \\
\hline & & & & Stroop & \\
\hline & & & RMSSD, HF & Letter Digit Substitution Task & \\
\hline Williams et al. & 2016 & $104 / 19.25 \pm 1.43$ healthy & HF, RMSSD & Modified flanker test & $\uparrow$ \\
\hline Yang et al. & 2008 & $\begin{array}{l}63 \text { men/78.3 } \pm 3.9 \\
\text { healthy }\end{array}$ & RMSSD & CASI & $\uparrow$ \\
\hline
\end{tabular}

Note. AD - Alzheimer's disease; CASI - Cognitive Abilities Screening Instrument; COPD - hypoxemic chronic obstructive pulmonary disease; CPT - continuous performance test; DGT-BACK - digit span raw score; CG - control group; DIGVIG - digit vigilance; HF - power in high frequency range; $\mathrm{MCl}$ - mild cognitive impaired; MOCA - Montreal Cognitive Assessment; nu - normalized units; RMSSD - root of the mean square of the differences of successive intervals; SD - standard deviation; SGST - Stop \& Go Switch Task; SP-BACK - spatial span backward raw score; WCST - Wisconsin card sorting test; WMS - Wechsler Memory Scale. $\uparrow$ : significant positive correlation. $\leftrightarrow$ : no correlation detected. 


\section{Memory and learning}

Memory and learning seem hardly related to HRV parameters (Table 2). There are some studies that found no connections (Britton, et al., 2008; Incalzi, et al., 2009; Jennings, et al., 2015; Nicolini, et al., 2014; Stenfors, et al., 2016). Only one study detected correlations: Nonogaki et al. (2017) showed that the Wechsler Memory Tasks were related to HF. There was a trend for HF in the verbal SRT (Selective Reminding Test) (Shah, et al., 2011).

\section{Language ability}

Language abilities were assessed in four studies (Table 3). Two of them showed no correlations (Britton, et al., 2008; Incalzi, et al., 2009). In the study by Britton et al. (2008), HF of 5375 subjects with a mean age of 55 years was measured. The study of Incalzi et al. (2009) measured patients with hypoxemic chronic obstructive pulmonary disease (COPD) and used two language tasks. Frequencydomain parameters did not correlate with task performance. But the language part of two cognitive test batteries showed correlations with HF: MOCA (Montreal Cognitive Assessment) (Frewen, et al., 2013) and CASI (Yang, et al., 2008). In both studies, healthy elderly people were tested.

\section{Visuospatial functioning}

The relationship between HRV and visuospatial functioning was investigated in three studies (Table 4). Frewen et al. (2013) found no association in 4763 healthy elderly subjects. Nicolini et al. (2014) found no correlation either. Here, healthy elderly people and subjects with MCI (mild cognitive impairment) were investigated. On the other hand, Incalzi et al. (2009) found a connection with frequency-domain

Table 2. HRV parameters and memory and learning

\begin{tabular}{|c|c|c|c|c|c|}
\hline Author & Year & $\begin{array}{l}\text { Participants (numberl } \\
\text { age in } M \pm S D \text { orland } \\
\text { range in years) }\end{array}$ & HRV parameters & Cognitive task & Result \\
\hline Britton et al. & 2008 & $\begin{array}{l}\text { 5375/55.5 (men), } 55.8 \\
\text { (women) }\end{array}$ & $\mathrm{HF}$ & 20 -word free recall test & $\leftrightarrow$ \\
\hline \multirow[t]{2}{*}{ Incalzi et al. } & \multirow[t]{2}{*}{2009} & \multirow[t]{2}{*}{ 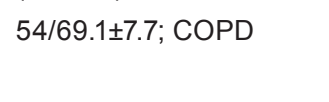 } & \multirow[t]{2}{*}{ HF nu } & Rey auditory 15 -word learning test & $\leftrightarrow$ \\
\hline & & & & Immediate visual memory & $\leftrightarrow$ \\
\hline Jennings et al. & 2015 & $440 / 43 \pm 7.3$; healthy & $\mathrm{HF}$ & 4WRD-STM & $\leftrightarrow$ \\
\hline Nicolini et al. & 2014 & $80 / 78.6 ; 40 \mathrm{MCl}, 40 \mathrm{CG}$ & HF nu & Digit Span Forward & $\leftrightarrow$ \\
\hline Nonogaki et al. & 2017 & 78/77.1 $\pm 6.2 ; A D$ & $\mathrm{HF}$ & Wechsler Memory & $\uparrow$ \\
\hline \multirow[t]{2}{*}{ Shah et al. } & \multirow[t]{2}{*}{2011} & \multirow[t]{2}{*}{416 men twins $/ 55.1 \pm 2.9$} & \multirow[t]{2}{*}{$\mathrm{HF}$} & SRT & $\leftrightarrow$ \\
\hline & & & & Visual SRT & $\leftrightarrow$ \\
\hline Stenfors et al. & 2016 & $\begin{array}{l}\text { 119/47.98 } \pm 10.49 ; 25-66 \text {; } \\
\text { healthy }\end{array}$ & RMSSD, HF & Reading Span Task & $\leftrightarrow$ \\
\hline
\end{tabular}

Note. 4WRD-STM - Four-Word Memory Test; CASI - Cognitive Abilities Screening Instrument; CG - control group; COPD - hypoxemic chronic obstructive pulmonary disease; $\mathrm{HF}$ - power in high frequency range; $\mathrm{MCl}$ - mild cognitive impaired; nu - normalized units; RMSSD - root of the mean square of the differences of successive intervals; SRT - Verbal Selective Reminding Test. $\uparrow:$ significant positive correlation. $\leftrightarrow$ : no correlation detected.

Table 3. HRV parameters and language ability

\begin{tabular}{|c|c|c|c|c|c|}
\hline Author & Year & $\begin{array}{l}\text { Participants (number/ } \\
\text { age in } M \pm S D \text { or/and } \\
\text { range in years) }\end{array}$ & HRV parameters & Cognitive task & Result \\
\hline \multirow[t]{3}{*}{ Britton et al. } & 2008 & $\begin{array}{l}\text { 5375/55.5 (men), } 55.8 \\
\text { (women); healthy }\end{array}$ & $\mathrm{HF}$ & Mill Hill Vocabulary Test & $\leftrightarrow$ \\
\hline & & & & Phonemic fluency & $\leftrightarrow$ \\
\hline & & & & Semantic fluency & $\leftrightarrow$ \\
\hline Frewen et al. & 2013 & $4763 / 61,7 \pm 8.3$; healthy & $\mathrm{HF}$ & MOCA & $\uparrow$ \\
\hline \multirow[t]{2}{*}{ Incalzi et al. } & 2009 & 54/69.1 $\pm 7.7 ;$ COPD & HF nu & Verbal fluency test & $\leftrightarrow$ \\
\hline & & & & Sentence construction & $\leftrightarrow$ \\
\hline Yang et al. & 2008 & $\begin{array}{l}63 \text { men/78.3 } \pm 3.9 \text {; } \\
\text { healthy }\end{array}$ & $\mathrm{HF}$ & CASI & $\uparrow$ \\
\hline
\end{tabular}

Note. CASI - Cognitive Abilities Screening Instrument; COPD - hypoxemic chronic obstructive pulmonary disease; HF - power in high frequency range; MOCA - Montreal Cognitive Assessment; nu - normalized units; SD - standard deviation. $\uparrow$ : significant positive correlation. $\leftrightarrow$ : no correlation detected. 
HRV. Regarding visuospatial functioning, there are too little studies to make any conclusions.

\section{Processing speed}

Six studies investigating the relationship between processing speed and HRV (Table 5) were identified. In none of them significant correlations between vagally mediated HRV and processing speed were detected. Specifically, there was no correlation with the non-executive part of CPT (Hansen, et al., 2003, 2004, 2009), Trail-Making Test A (Nicolini, et al., 2014; Stenfors, et al., 2016), and backward counting (Kimhy, et al., 2013).

\section{Global cognitive functioning}

Some authors did not differentiate between single cognitive functions but used general cognitive tests or presented only the total score of a cognitive test battery (Table 6). The MMSE (Mini Mental Status Examination) is one of the most widespread cognitive test batteries. One study showed positive associations between the total score of MMSE and RMSSD (Kim, et al., 2006). Associations with the frequency-domain parameters were found in the same study and a non-significant trend in the study by Mellingsæter, Wyller, Ranhoff, Bogdanovic, and Wyller (2015), but not in the study by Allan et al.

Table 4. HRV parameters and visuospatial functioning

\begin{tabular}{|c|c|c|c|c|c|}
\hline Author & Year & $\begin{array}{l}\text { Participants (number/ } \\
\text { age in } M \pm S D \text { or/and } \\
\text { range in years) }\end{array}$ & HRV parameters & Cognitive task & Result \\
\hline Frewen et al. & 2013 & $4763 / 61,7 \pm 8.3 ;$ healthy & $\mathrm{HF}$ & MOCA & $\leftrightarrow$ \\
\hline Incalzi et al. & 2009 & 54/69.1 $\pm 7.7 ;$ COPD & HF nu & CDL & $\downarrow$ \\
\hline Nicolini et al. & 2014 & $80 / 78.6 ; 40 \mathrm{MCl}, 40 \mathrm{CG}$ & HF nu & $\begin{array}{l}\text { Rey-Osterrieth complex figure- } \\
\text { delayed recall }\end{array}$ & $\leftrightarrow$ \\
\hline
\end{tabular}

Note. CDL - copying of drawing landmarks; CG - control group; COPD - hypoxemic chronic obstructive pulmonary disease; HF power in high frequency range; $\mathrm{MCl}$ - mild cognitive impaired; MMSE - Mini-Mental-Status-Examination; MOCA - Montreal Cognitive Assessment; nu - normalized units; SD - standard deviation. $\uparrow:$ significant positive correlation. $\leftrightarrow$ : no correlation detected.

Table 5. HRV parameters and processing speed

\begin{tabular}{|c|c|c|c|c|c|}
\hline Author & Year & $\begin{array}{l}\text { Participants (number/ } \\
\text { age in } M \pm S D \text { orland } \\
\text { range in years) }\end{array}$ & HRV parameters & Cognitive task & Result \\
\hline Hansen et al. & 2004 & 37 men/19.1; healthy & HF & CPT & $\leftrightarrow$ \\
\hline Hansen et al. & 2003 & 53 men/23; healthy & RMSSD & СРТ & $\leftrightarrow$ \\
\hline Hansen et al. & 2009 & $65 / 23.1$; healthy & HF & СРТ & $\leftrightarrow$ \\
\hline Kimhy et al. & 2013 & $817 / 57.11 \pm 11.15$; healthy & $\mathrm{HF}$ & Backward counting & $\leftrightarrow$ \\
\hline Nicolini et al. & 2014 & $80 / 78.6 ; 40 \mathrm{MCl}, 40 \mathrm{CG}$ & HF nu & Trail-Making Test A & $\leftrightarrow$ \\
\hline Stenfors et al. & 2016 & $\begin{array}{l}\text { 119/47.98 } \pm 10.49 ; 25-66 \text {; } \\
\text { healthy }\end{array}$ & RMSSD, HF & Trail-Making Test A & $\leftrightarrow$ \\
\hline
\end{tabular}

Note. CG - control group; CPT - continuous performance test; HF - power in high frequency range; $\mathrm{MCl}$ - mild cognitive impaired; nu - normalized units; RMSSD - root of the mean square of the differences of successive intervals; SD - standard deviation. $\uparrow$ : significant positive correlation. $\leftrightarrow$ : no correlation detected.

Table 6. HRV parameters and global cognitive function

\begin{tabular}{|c|c|c|c|c|c|}
\hline Author & Year & $\begin{array}{l}\text { Participants (number/age in } M \pm S D \text { orl } \\
\text { and range in years) }\end{array}$ & HRV parameters & Cognitive task & Result \\
\hline Allan et al. & 2005 & $114 />65 ; 80$ healthy, 14 AD, 20 VAD & $\mathrm{HF}$ & MMSE & $\leftrightarrow$ \\
\hline Allan et al. & 2007 & $\begin{array}{l}177 />65 ; 39 \mathrm{AD}, 30 \mathrm{VAD}, 30 \mathrm{DLB}, 40 \mathrm{PDD} \text {, } \\
38 \mathrm{CG}\end{array}$ & $\mathrm{HF}$ & CAMCOG & $\uparrow$ \\
\hline Kim et al. & 2006 & 311 women/65-85 disabled & HF, RMSSD & MMSE & $\uparrow$ \\
\hline $\begin{array}{l}\text { Mellingsæter } \\
\text { et al. }\end{array}$ & 2015 & $62 / 14 \mathrm{AD}$ or $\mathrm{MCl}(73.6), 48 \mathrm{CG}(72) ;>65$ & $\mathrm{HF}$ & MMSE & $\uparrow$ \\
\hline
\end{tabular}

Note. AD - Alzheimer's disease; CAMCOG - Cambridge Examination for Mental Disorders in the Elderly; CG - control group; DLB - dementia with Lewy bodies; HF - power in high frequency range; $\mathrm{MCl}$ - mild cognitive impaired; MMSE - Mini-Mental-StatusExamination; PDD - Parkinson's disease dementia; RMSSD - root of the mean square of the differences of successive intervals; SD - standard deviation; VAD - vascular Alzheimer's disease. $\uparrow$ : significant positive correlation. $\leftrightarrow$ : no correlation detected. 
(2005). Allan et al. (2007) detected correlations between HF and the total score of the test battery CAMCOG (Cambridge Examination for Mental Disorders in the Elderly) in 177 elderly people.

\section{Discussion and conclusion}

The purpose of the current review was to summarize the existing literature exploring the cross-sectional correlation between resting HRV and cognitive functions. Possible reasons for both the detected and not detected correlations are presented.

\section{HRV and executive functions}

Executive functions comprise working memory, inhibition and cognitive flexibility (Albinet, et al., 2016). Our results showed a partly relation between executive performances and HRV levels. Some studies detect associations with the vagally mediated HRV parameters HF or RMSSD (Albinet, et al., 2010; Hansen, et al., 2003, 2004, 2009; Hovland, et al., 20012; Jennings, et al., 2015; Kemp, et al., 2016; Luque-Casado, et al., 2013; Williams, et al., 2016; Yang, et al., 2008), but others did not find any associations (Alderman \& Olson, 2014; Britton, et al., 2008; Duschek, et al., 2009; Frewen, et al., 2013; Giuliano, et al., 2017; Incalzi, et al., 2009; Kimhy, et al., 2013; Mathewson, et al., 2010; Nicolini, et al., 2014; Nonogaki, et al., 2017; Stenfors, et al., 2016).

Hansen et al. (2003) justified their positive results through the association between HRV and "efficient attentional regulation and greater ability to inhibit pre-potent but inappropriate responses". This statement underpins the supposed relationship between HRV and the prefrontal cortex and explains the positive association between vagally mediated HRV and executive function tasks like the task Stroop. Hansen et al. (2004) supposed that the positive relationship was the result of the increased efficiency of prefrontal neural function achieved through aerobic training. In particular, physical training leads to cardiovascular improvements and increased cerebral blood flow, which improves prefrontal functioning.

One explanation for the lack of correlations in other studies could be the big number of factors playing their roles in the relationship between cognitive performance and HRV (Alderman \& Olson, 2014). Kimhy et al. (2013) mentioned a limited instrument sensitivity of the working memory task as one reason for the lack of correlation. Britton et al. (2008) stated that their test battery did not assess executive functions in detail. Nonogaki et al. (2017) called the old age of their subjects to account for the lack of correlations and maybe dementia hid possible correlations. However, Holzman and Bridgett (2017) noted that the relation between HRV and top-down self-regulation is stronger in older individuals. Nicolini et al. (2014) stated that autonomic dysfunction is revealed only when the autonomic system is challenged by changing from the supine position to the standing one. In summary, the results demonstrated a tendency of positive correlations between typical executive function tasks (Stroop and 2-back) and vagally mediated HRV.

\section{HRV and memory learning}

Memory was positively associated with vagally mediated HRV in only one study with AD patients (Nonogaki, et al., 2017). The authors argued that some parts of the brain (insular cortex, amygdala, hypothalamus, and nucleus tractus solitarius) regulate cardiac autonomic function and interact with the medial temporal lobes. These regions are responsible for memorizing things but get impaired in case of a central autonomic dysfunction, which is the case in AD. Karlamangla et al. (2014) confirmed this by revealing a positive relationship between cardiovascular function and memory. Shah et al. (2011) mentioned an impaired baroreflex as a possible mechanism because it is responsible for the cerebral blood flow and is an important component of the autonomic nervous system. In summary, the results indicate a weak connection between memory and HRV.

\section{HRV and language ability}

Language ability includes comprehension and word finding (Levy, 1994). In two studies, positive correlations with HF were detected (Frewen, et al., 2013; Yang, et al., 2008). Frewen et al. (2013) mentioned the fornix, a connective part for the hippocampus in the brain, as a possible location underlying the association between HRV and cognition. Moreover, the cholinergic anti-inflammatory pathway could explain this relationship as well.

\section{HRV and visuospatial functioning}

Regarding the visuospatial functioning, one study detected significant correlations. Incalzi et al. (2009) explained their results with a dysfunction of the right insular in COPD patients and concluded that "neurogenic heart diseases" are an expression of cardiac abnormalities related to problems in the brain-heart connection.

\section{HRV and processing speed}

Processing speed was added as a non-executive function, measuring the reaction time in simple choice tasks that do not require challenging cognitive functions. None of the six relevant studies found significant correlations. There was at least a trend in the Trail-Making Test A (Nicolini, et al., 2014; Stenfors, et al., 2016). Stenfors et al. (2016) suggested using the parameter QTVI (QT variability index) because it showed the best correlations 
between HRV and cognitive functions. In the study of Hansen et al. (2004), curiously, the low HRV group had faster reaction times compared to the high HRV group in a non-executive task.

\section{HRV and global cognitive functions}

In three out of four studies, positive correlations between total cognitive scores and HRV were detected. HF correlated significantly with the scores of the MMSE (Kim, et al., 2006; Mellingsæter, et al., 2015) and the CAMCOG (Allan, et al., 2007). Additionally, RMSSD showed a positive correlation with the MMSE (Kim, et al., 2006). These results implicate that HRV reflects broad cognitive functions and not only specific ones.

\section{Mechanism linking HRV and cognitive function}

The role of the HRV as a possible biomarker of the top-down self-regulatory mechanism was well discussed in a recent meta-analysis (Holzman \& Bridgett, 2017). The present systematic review focuses on the cognitive part of self-regulation. Thayer et al. (2009) suggested that HRV served as an index of the functional capacity of brain structures "that support the effective and efficient performance of cognitive executive-function tasks including working memory and inhibitory control".

Two theories are the basis for the considered relation between HRV and top-down self-regulation that comprises cognitive functions: the Neurovisceral Integration Model (Thayer, et al., 2009) and the Polyvagal Theory (Porges, 2003). Both approaches stated a moderating role of the parasympathetic-mediated nervous system. The prefrontal cortex is supposed to play an important role thereby. This part of the brain forms together with cortical and subcortical brain structures an interconnected network and controls the heart rate being also responsible for the cognitive regulation (Thayer \& Lane, 2009). Thus, a declining prefrontal activity leads to an increased sympathetic activity, heart rate and hence reduced HRV (Thayer \& Lane, 2009). This inhibition of prefrontal activity and pronounced activation of the sympathetic system was beneficial for human survival in ancient times. However, nowadays, a tonic inhibition of the prefrontal cortex and a permanent sympathetic state are contradictory to adaptation and self-regulatory processes (McCraty \& Shaffer, 2015). Thus, an optimal level of vagally mediated HRV is necessary for adaptation and cognitive functions.

The link between prefrontal cortex and cardiac system was mentioned in some studies and called upon as one explanation for the correlation between HRV and cognition. The functional state of the cardiac system depends on the level of brain perfusion that can be improved through aerobic training
(Mahinrad, et al., 2016). Hansen et al. (2004) argued that the relationship is "mediated by a common set of neural circuits, the efficient functioning of which have inhibitory processes". Mahinrad et al. (2016) considered an autonomic dysfunction caused by dysregulation in cerebral perfusion as the link between low HRV and cognitive impairment. External factors like cardiovascular risk factors could be responsible for the association as well. Further, HRV could reflect an early manifestation of brain damage and future cardiovascular events which lead to cognitive decline. The baroreflex is another possible explanation for the relation between HRV and cognition (Kim, et al., 2006). It ensures proper blood flow through all organs and is regarded an indicator for the autonomic nervous system. High blood pressure variability is a sign of baroreflex disturbances and is associated with cognitive impairment (Meyer, et al., 2017). The relationship between blood pressure regulation and dementia was recently reviewed by O'Callaghan and Kenny (2016). They have described the phenomenon of Neurocardiovascular Instability (NCVI), which affects heart rate behaviour through abnormal neural control and is more prevalent in patients with MCI or AD. However, the direction of causality is still unclear. Another reason for the relationship may be a dysfunction of the cholinergic system, which impairs cognitive and parasympathetic system function (Frewen, et al., 2013; Yang, et al., 2008). Incalzi et al. (2009) have mentioned a dysfunction of the right insular as an additional reason for the relationship because the insular modulates sympathetic tone and is involved in integrating cognitive and emotional aspects. Allan et al. (2007) listed autonomic neuropathy as a possible explanation because frequency-domain HRV was lower in patients with Parkinson's disease, who showed higher prevalence of autonomic neuropathy.

\section{Limitations}

Some limitations have to be mentioned. First, the studies used different HRV measurement techniques and durations of recording the HRV. This could lead to inconsistency in the results. Second, different samples in terms of age-range and state of health were compared. This was done to offer a variety of studies with different sample characteristics. Otherwise, the number of studies would have been much smaller. And third, our classification of cognitive functions was restricted in the way that a lot of different tasks were used and therefore it was difficult to classify and compare them.

\section{Conclusions}

A summary of studies investigating the association between resting-state HRV and cognitive performance was provided. Theoretical models 
proposed a positive connection between HRV and cognitive performance through the link between cardiovascular regulating processes in the brain and cognition regulating processes located, especially, in the prefrontal cortex (McCraty, et al., 2015; Shaffer, et al., 2014; Thayer, et al., 2009). Some studies could confirm this relationship (e.g., Albinet, et al., 2010; Hansen, et al., 2009; Hovland, et al., 2012; Yang, et al., 2008), but others could not (e.g., Britton, et al., 2008; Nicolini, et al., 2014). The lack of a uniform measurement procedure, different cognitive tasks, and different sample characteristics (age, disorders) are possible reasons for the lack of a stronger relation. To take a step forward in diagnosing and preventing dementia, longitudinal studies are recommended to evaluate $\mathrm{HRV}$ as an indicator of cognitive impairment and to investigate the development of and interaction between HRV and cognitive performance throughout life.

\section{References}

Albinet, C.T., Abou-Dest, A., André, N., \& Audiffren, M. (2016). Executive functions improvement following a 5 -month aquaerobics program in older adults: Role of cardiac vagal control in inhibition performance. Biological Psychology, 115, 69-77. doi: 10.1016/j.biopsycho.2016.01.010

Albinet, C.T., Boucard, G., Bouquet, C., \& Audiffren, M. (2010). Increased heart rate variability and executive performance after aerobic training in the elderly. European Journal of Applied Physiology, 109, 617-624. doi: 10.1007/s00421-010-1393-y

Alderman, B.L., \& Olson, R.L. (2014). The relation of aerobic fitness to cognitive control and heart rate variability: A neurovisceral integration study. Biological Psychology, 99, 26-33. doi: 10.1016/j.biopsycho.2014.02.007

Allan, L.M., Ballard, C.G., Allen, J., Murray, A., Davidson, A.W., McKeith, I.G., \& Kenny, R.A. (2007). Autonomic dysfunction in dementia. Journal of Neurology, Neurosurgery, and Psychiatry, 78(7), 671-677. doi: 10.1136/ jnnp.2006.102343

Allan, L.M., Kerr, S.R.J., Ballard, C.G., Allen, J., Murray, A., McLaren, A.T., \& Kenny, R.A. (2005). Autonomic function assessed by heart rate variability is normal in Alzheimer's disease and vascular dementia. Dementia and Geriatric Cognitive Disorders, 19, 140-144. doi: 10.1159/000082885

Almeida-Santos, M.A., Barreto-Filho, J.A., Oliveira, J.L.M., Reis, F.P., da Cunha Oliveira, C.C., \& Sousa, A.C. (2016). Aging, heart rate variability and patterns of autonomic regulation of the heart. Archives of Gerontology and Geriatrics, 63, 1-8. doi: 10.1016/j.archger.2015.11.011

Billman, G.E. (2013). The LF/HF ratio does not accurately measure cardiac sympatho-vagal balance. Frontiers in Physiology, 4(26). doi: 10.3389/fphys.2013.00026

Billman, G.E. (2011). Heart rate variability - A historical perspective. Frontiers in Physiology, 2(86). doi: 10.3389/ fphys.2013.00026

Blondell, S.J., Hammersley-Mather, R., \& Veerman, J.L. (2014). Does physical activity prevent cognitive decline and dementia? A systematic review and meta-analysis of longitudinal studies. BMC Public Health, 14(510). doi: $10.1186 / 1471-2458-14-510$

Britton, A., Singh-Manoux, A., Hnatkova, K., Malik, M., Marmot, M.G., \& Shipley, M. (2008). The association between heart rate variability and cognitive impairment in middle-aged men and women. Neuroepidemiology, 31(2), 115-121. doi: $10.1159 / 000148257$

Bugg, J.M., DeLosh, E.L., Davalos, D.B., \& Davis, H.P. (2007). Age differences in Stroop interference: Contributions of general slowing and task-specific deficits. Aging, Neuropsychology, and Cognition, 14(2), 155-167. doi: $10.1080 / 138255891007065$

Duschek, S., Muckenthaler, M., Werner, N., \& Reyes del Paso, G.A. (2009). Relationships between features of autonomic cardiovascular control and cognitive performance. Biological Psychology, 81(2), 110-117. doi: 10.1016/j. biopsycho.2009.03.003

Fatisson, J., Oswald, V., \& Lalonde, F. (2016). Influence diagram of physiological and environmental factors affecting heart rate variability: An extended literature overview. Heart International, 11(1), 32-40. doi: 10.5301/ heartint.5000232

Frewen, J., Finucane, C., Savva, G.M., Boyle, G., Coen, R.F., \& Kenny, R.A. (2013). Cognitive function is associated with impaired heart rate variability in ageing adults: The Irish longitudinal study on ageing wave one results. Clinical Autonomic Research, 23(6), 313-323. doi: 10.1007/s10286-013-0214-x

Giuliano, R.J., Gatzke-Kopp, L.M., Roos, L.E., \& Skowron, E.A. (2017). Resting sympathetic arousal moderates the association between parasympathetic reactivity and working memory performance in adults reporting high levels of life stress. Psychophysiology, 54(8), 1195-1208. doi: 10.1111/psyp.12872 
Hansen, A.L., Johnsen, B.H., Sollers III, J.J., Stenvik, K., \& Thayer, J.F. (2004). Heart rate variability and its relation to prefrontal cognitive function: The effects of training and detraining. European Journal of Applied Physiology, 93(3), 263-274. doi: 10.1007/s00421-004-1208-0

Hansen, A.L., Johnsen, B.H., \& Thayer, J.F. (2009). Relationship between heart rate variability and cognitive function during threat of shock. Anxiety, Stress and Coping, 22(1), 7-89. doi: 10.1080/10615800802272251

Hansen, A.L., Johnsen, B.H., \& Thayer, J.F. (2003). Vagal influence on working memory and attention. International Journal of Psychophysiology, 48(3), 263-274. doi: 10.1016/S0167-8760(03)00073-4

Holzman, J.B., \& Bridgett, D.J. (2017). Heart rate variability indices as bio-markers of top-down self-regulatory mechanisms: A meta-analytic review. Neuroscience and Biobehavioral Reviews, 74, 233-255. doi: 10.1016/j. neubiorev.2016.12.032

Hottenrott, K., Lauenroth, A., \& Schwesig, R. (2004). Einfluss eines achtwöchigen Walkingtrainings auf Herzfrequenz und die Herzfrequenzvariabilität bei über 60-jährigen. [Influence of an eight-week walking training in heart rate and heart rate variability in people above 60 years. In German.] In K. Hottenrot (Ed.), Herzfrequenzvariabilität im Fitness und Gesundheitssport [Heart rate variability in fitness and recreational sport] (pp. 191-197). Halle/ Saale: Czwalina.

Hovland, A., Pallesen, S., Hammar, Å., Hansen, A.L., Thayer, J.F., Tarvainen, M.P., \& Nordhus, I.H. (2012). The relationships among heart rate variability, executive functions, and clinical variables in patients with panic disorder. International Journal of Psychophysiology, 86(3), 269-275. doi: 10.1016/j.ijpsycho.2012.10.004

Incalzi, R.A, Corsonello, A., Trojano, L., Pedone, C., Acanfora, D., Spada, A., D’Addio, G., Maestri, R., Rengo, F., \& Rengo, G. (2009). Heart rate variability and drawing impairment in hypoxemic COPD. Brain and Cognition, 70(1), 163-170. doi: 10.1016/j.bandc.2009.01.010

Jandackova, V.K., Scholes, S., Britton, A., \& Steptoe, A. (2016). Are changes in heart rate variability in middle-aged and older people normative or caused by pathological conditions? Findings from a Large Population-Based Longitudinal Cohort Study. Journal of the American Heart Association, 5(2). doi: 10.1161/JAHA.115.002365

Jennings, J.R., Allen, B., Gianaros, P.J., Thayer, J.F., \& Manuck, S.B. (2015). Focusing neurovisceral integration: Cognition, heart rate variability, and cerebral blood flow. Psychophysiology, 52(2), 214-224. doi: 10.1111/ psyp.12319

Karlamangla, A.S., Miller-Martinez, D., Lachman, M.E., Tun, P.A., Koretz, B.K., \& Seeman, T.E. (2014). Biological correlates of adult cognition: Midlife in the United States (MIDUS). Neurobiology of Aging, 35(2), $387-394$. doi: 10.1016/j.neurobiolaging.2013.07.028

Kemp, A.H., Koenig, J., \& Thayer, J.F. (2017). From psychological moments to mortality: A multidisciplinary synthesis on heart rate variability spanning the continuum of time. Neuroscience and Biobehavioral Reviews, 83, 547-567. doi: 10.1016/j.neubiorev.2017.09.006

Kemp, A.H., López, S.R., Passos, V.M.A., Bittencourt, M.S., Dantas, E.M., Mill, J.G., Ribeiro, A.L.P., Thayer, J.F., Bensenor, I.M., \& Lotufo, P.A. (2016). Insulin resistance and carotid intima-media thickness mediate the association between resting-state heart rate variability and executive function: A path modelling study. Biological Psychology, 117, 216-224. doi: 10.1016/j.biopsycho.2016.04.006

Kim, D.H., Lipsitz, L.A., Ferrucci, L., Varadhan, R., Guralnik, J.M., Carlson, M.C., Fleisher, L.A., Fried, L.P., \& Chaves, P.H.M. (2006). Association between reduced heart rate variability and cognitive impairment in older disabled women in the community: Women's Health and Aging Study I. Journal of the American Geriatrics Society, 54(11), 1251-1757. doi: 10.1111/j.1532-5415.2006.00940.x

Kimhy, D., Crowley, O.V., McKinley, P.S., Burg, M.M., Lachman, M.E., Tun, P.A., Ryff, C.D., Seeman, T.E., \& Sloan, R.P. (2013). The association of cardiac vagal control and executive functioning - Findings from the MIDUS Study. Journal of Psychiatric Research, 47(5), 628-635. doi: 10.1016/j.jpsychires.2013.01.018

Levy, R.L. (1994). Aging-associated cognitive decline. International Psychogeriatrics, 6(1), 63-68.

Luque-Casado, A., Zabala, M., Morales, E., Mateo-March, M., \& Sanabria, D. (2013). Cognitive performance and heart rate variability: The influence of fitness level. PLoSOne, 8(2). doi: 10.1371/journal.pone.0056935

Mahinrad, S., Jukema, J.W., von Heemst, D., Macfarlane, P.W., Clark, E.N., de Craen, A.J.M., \& Sabayan, B. (2013). 10 -second heart rate variability and cognitive function in old age. Neurology, 86(12), 1120-1127. doi: 10.1212/ WNL.0000000000002499

Makivić, B., Nikić, M.D., \& Willis, M.S. (2013). Heart rate variability (HRV) as a tool for diagnostic and monitoring performance in sport and physical activities. Journal of Exercise Physiology, 16(3), 103-131.

Mathewson, K.J., Jetha, M.K., Drmic, I.E., Bryson, S.E., Goldberg, J.O., Hall, G.B., Santesso, D.L., Segalowitz, S.J., \& Schmidt, L.A. (2010). Autonomic predictors of Stroop performance in young and middle-aged adults. International Journal of Psychophysiology, 76(3), 123-129. doi:10.1016/j.ijpsycho.2010.02.007

McCraty, R., \& Shaffer, F. (2015). Heart rate variability: New perspectives on physiological mechanisms, assessment of self-regulatory capacity, and health risk. Global Advances in Health and Medicine, 4(1), 46-61. doi: 10.7453/ gahmj.2014.073

Mellingsæter, M.R., Wyller, T.B., Ranhoff, A.H., Bogdanovic, N., \& Wyller, V.B. (2015). Reduced sympathetic response to head-up tilt in subjects with mild cognitive impairment or mild Alzheimer's dementia. Dementia and Geriatric Cognitive Disorders Extra, 5(1), 107-115. doi: 10.1159/000375297 
Melo, R.C., Santos, M.D.B., Silva, E., Quitério, R.J., Moreno, M.A., Reis, M.S., Verzola, I.A., Oliveira, L., Martins, L.E.B., Gallo-Junior, L., \& Catai, A.M. (2005). Effects of age and physical activity on the autonomic control of heart rate in healthy men. Brazilian Journal of Medical and Biological Research, 38(9), 1331-1338. doi: / S0100-879X2005000900007

Meyer, M.L., Palta, P., Tanaka, H., Deal, J.A., Wright, J., Knopman, D.S., Griswold, M.E., Hosley, T.H., \& Heiss, G. (2017). Association of central arterial stiffness and pressure pulsatility with mild cognitive impairment and dementia. The Atherosclerosis Risk in Communities Study - Neurocognitive Study (ARIC-NCS). Journal of Alzheimer's Disease, 57(1), 195-204. doi: 10.3233/JAD-161041

Moher, D., Liberati, A., Tetzlaff, J., \& Altman, D.G. (2009). Preferred reporting items for systematic reviews and meta-analyses: The PRISMA Statement. Journal of Clinical Epidemiology, 62(10), 1006-1012. doi: 10.1016/j. jclinepi.2009.06.005

Nicolini, P., Ciull, M.M., Malfatto, G., Abbate, C., Mari, D., Rossi, P.D., Pettenuzzo, E., Magrini, F., Consonni, D., \& Lombardi, F. (2014). Autonomic dysfunction in mild cognitive impairment: Evidence from Power Spectral Analysis of Heart Rate Variability in a Cross-Sectional Case-Control Study. PLoS One, 9(5). doi: 10.1371/ journal.pone.0096656

Nonogaki, Z., Umegaki, H., Makino, T., Suzuki, Y., \& Kuzuya, M. (2017). Relationship between cardiac autonomic function and cognitive function in Alzheimer's disease. Geriatrics and Gerontology International, 17(1), 92-98. doi: $10.1111 /$ ggi.12679

O’Callaghan, S., \& Kenny, R.A. (2016). Neurocardiovascular instability and cognition. Yale Journal of Biology and Medicine, 89(1), 59-72.

Porges, S.W. (2003). The Polyvagal Theory: Phylogenetic contributions to social behavior. Physiology and Behavior, 79(3), 503-513.

Quintana, D.S, Guastella, A.J., Outhred, T., Hickie, I.B., \& Kemp, A.H. (2012). Heart rate variability is associated with emotion recognition: Direct evidence for a relationship between the autonomic nervous system and social cognition. International Journal of Psychophysiology, 86(2), 168-172. doi: 10.1016/j.ijpsycho.2012.08.012

Shaffer, F., McCraty, R., \& Zerr, C.L. (2014). A healthy heart is not a metronome: An integrative review of the heart's anatomy and heart rate variability. Frontiers in Psychology, 5(1040). doi: 10.3389/fpsyg.2014.01040

Shah, A.J., Su, S., Veledar, E., Bremner, J.D., Goldstein, F.C., Lampert, R., Goldberg, J., \& Vaccarino, V. (2011). Is heart rate variability related to memory performance in middle aged men? Psychosomatic Medicine, 73(6), 475-482. doi: 10.1097/PSY.0b013e3182227d6a

Singh, J.P., Larson, M.G., O’Donnell, C.J., Tsuji, H., Evans, J.C., \& Levy, D. (2001). Heritability of heart rate variability: The Framingham Heart Study. Circulation, 99(17), 2251-2254.

Stein, P.K., Domitrovich, P.P., Hui, N., Rautaharju, P., \& Gottdiener, J. (2005). Sometimes higher heart rate variability is not better heart rate variability. Journal of Cardiovascular Electrophysiology, 16(9), 954-959. doi: 10.1111/j.15408167.2005.40788.x

Stein, P.K., \& Kleiger, R.E. (1999). Insights from the study of heart rate variability. Annual Review of Medicine, 50, 249-261.

Stenfors, C.U.D., Hanson, L.M., Theorell, T., \& Osika, W.S. (2016). Executive cognitive functioning and cardiovascular autonomic regulation in a population-based sample of working adults. Frontiers in Psychology, 7(1536). doi: 10.3389/fpsyg.2016.01536

Sztajel, J. (2004). Heart rate variability: A noninvasive electrocardiographic method to measure the autonomic nervous system. Swiss Medical Weekly, 134(35-36), 514-522. doi: 2004/35/smw-10321

Thayer, J.F., Åhs, F., Fredrikson, M., Sollers III, J.J., \& Wager, T.D. (2012). A meta-analysis of heart rate variability and neuroimaging studies: Implications for heart rate variability as a marker of stress and health. Neuroscience and Biobehavioral Reviews, 36(2), 747-756. doi: 10.1016/j.neubiorev.2011.11.009

Thayer, J.F., Hansen, A.L., Saus-Rose, E., \& Johnsen, B.H. (2009). Heart rate variability, prefrontal neural function, and cognitive performance: The neurovisceral integration perspective on self-regulation, adaptation, and health. Annals of Behavioral Medicine, 37(2), 141-153. doi: 10.1007/s12160-009-9101-z

Thayer, J.F., \& Lane, R.D. (2009). Claude Bernard and the heart-brain connection: Further elaboration of a model of neurovisceral integration. Neuroscience and Biobehavioral Reviews, 33(2), 81-88. doi: 10.1016/j. neubiorev.2008.08.004

Thayer, J.F., Yamamoto, S.S., \& Brosschot, J.F. (2010). The relationship of autonomic imbalance, heart rate variability and cardiovascular disease risk factors. International Journal of Cardiology, 141(2), 122-131. doi: 10.1016/j. ijcard.2009.09.543

Togo, F., \& Takahashi, M. (2009). Heart rate variability in occupational health - A systematic review. Industrial Health, 47(6), 589-602.

Valentini, M., \& Parati, G. (2009). Variables influencing heart rate. Progress in Cardiovascular Diseases, 52(1), 11-19. doi: 10.1016/j.pcad.2009.05.004.

Wild-Wall, N.N., Falkenstein, M., \& Gajewski, P.D. (2011). Age-related differences in working memory performance in a 2-back task. Frontiers in Psychology, 186(2). doi: 10.3389/fpsyg.2011.00186. 
Williams, D.P., Thayer, J.F., \& Koenig, J. (2016). Resting cardiac vagal tone predicts intraindividual reaction time variability during an attention task in a sample of young and healthy adults. Psychophysiology, 53(12), 18431851. doi: $10.1111 /$ psyp.12739

Yang, A.C., Tsai, S.J., Hong, C.J., Yang, C.H., Hsieh, C.H., \& Liu, M.E. (2008). Association between heart rate variability and cognitive function in elderly community-dwelling men without dementia: A preliminary report. Journal of the American Geriatrics Society, 56(5), 958-960. doi: 10.1111/j.1532-5415.2008.01662.x

Submitted: July 18, 2018

Accepted: May 5, 2019

Published Online First: April 30, 2020

Correspondence to:

Bernhard Grässler, Ph.D.

Otto-von-Guericke-University Magdeburg,

Department of Sport Science

Germany

Phone: 00493616756682

Fax: 00493616746754

E-mail: bernhard.graesler@ovgu.de 